Kocaeli Journal of Science and Engineering

\title{
Model Updating of a Euler-Bernoulli Beam Using the Response Method
}

\author{
Cevher Yusuf INAN iD Akın OKTAV ${ }^{2}$ \\ ${ }^{1}$ Abdullah Gul University, Mechanical Engineering Departmen, Kayseri, Turkey, ORCID: 0000-0002-8818-4750 \\ ${ }_{2}^{2}$ Abdullah Gul University, Mechanical Engineering Departmen, Kayseri, Turkey, ORCID: 0000-0001-5983-3953
}

Article Info

Research paper

Received : July 11, 2020

Accepted : : December 24, 2020

Keywords

Frequency Response Function,

Model Updating,

Structural Damping,

Viscous Damping

\section{Abstract}

In this study, the computational model is updated using an analytical model instead of an experimental one. Continuous and discrete parameter models of a Euler-Bernoulli beam are constructed analytically and computationally. To construct the computational models, Ansys ${ }^{\mathrm{TM}}$ software is employed, and 1-D beam elements are chosen to get the finite element model of a cantilever beam. To get analytical solutions for the continuous and discrete parameter models, a state-space representation is employed. In the first step, only mass and stiffness matrices are considered to model the beam. Eigenfrequencies and eigenvectors of the beam are calculated. The analytical and computational eigenfrequencies of continuous and discrete parameter models are compared. In the seconds step, non-proportional viscous damping and non-proportional structural damping matrices are introduced into the analytical discrete parameter model. Then, the frequency response functions of the model are generated. The damping matrices are identified using the generated frequency response functions. The damping matrices used in the analytical model, and the damping matrices identified using the frequency response functions are compared. It is observed that the assigned damping matrices and the identified damping matrices are identical, which shows that the computational model can be accurately updated provided the FRFs are available.

\section{Introduction}

Deterministic element-based methods are widely used for modeling in structural dynamics; the most common one is the finite element method (FEM). Dynamic systems are mathematically expressed by mass, stiffness, and damping matrices. Finite element packages can populate mass and stiffness matrices using the data given in the preprocessing step such as, geometry and material properties of the structure. At this step, the model does not have a damping property, yet. Based on the Rayleigh damping model, a proportional damping matrix can be created using mass and stiffness matrices. This damping matrix does not reflect the actual physical behavior. For this reason, errors may occur in the final updated model. The error rate increases as the degrees of freedom (dof) of the dynamic model increase.

\footnotetext{
* Corresponding Author: akin.oktav@agu.edu.tr
}

Model updating has been developed with many different methods proposed since 1980s. Mottershead and Friswell systematically summarized the classical model update theory [1]. There are different approaches to model updating in the literature. The relevant methods are based on a direct algorithm or an iteration algorithm. System parameter-based methods have a more apparent physical meaning and better computing performance than matrixbased methods. Response data-based methods are becoming more common as there is an advantage to avoid errors arising from mode identification. Due to the difficulty of modeling damping and measuring the dynamic properties, there is incompleteness in updating the damping values in existing model update methods.

The mass matrix of a structure can accurately be estimated by finite element model, but there may be errors in the stiffness matrix. For this reason, it is stated that the stiffness matrix should be updated using an appropriate method [2]. A method that reduces the norm of the difference between the computational and the experimental modes is proposed [3]. It states that the mass and the 
stiffness matrices should be evaluated simultaneously, when updating a model. The model update process would be more successful, if performed with the experimental model [4]. In another study, it is stated that mass, stiffness, and damping matrices should be updated simultaneously with the experimental data [5]. In a study held in 2012, uncertainties in experimental data were evaluated [6]. These studies mentioned in the literature are direct methods.

Iteration methods are another topic in model update. It is the method of matching the modal matrices obtained with computational models using experimental data and constructing the updated model. In the literature, iteration is performed using frequency response functions (FRFs) measured experimentally, which is known as the response function method [7]. The response function method is expanded to update the proportional viscous and the proportional structural damping matrices [8]. In another study, the differences between the computational data and the experimental data are minimized by the so-called Taguchi method [9]. Sipple and Sanayei proposed a new approach to solve the inverse problem [10]. They used a new frequency response function-based model update method by employing numerical sensitivity, instead of analytical sensitivity [10]. It is proposed that the model update should be performed with undamped frequency response functions [11]. Researchers developed a new method to define the structural damping matrix which can be used in simple structures as the procedure requires exact measurements [12]. The error margin in the model update approach is computed by Matta and Stefano [13] using the experimental data of a large building. Damping is a crucial parameter in model updating studies. There are studies which compare different damping models in the literature [14][15].

In the response method, frequency response functions are used to quantify the dynamic characteristics of the model [16-18]. Natke has summarized the model updating methods based on the transfer character [19]. In another study, residues in resonance and anti-resonance are taken as a reference to update a damped model. Lu and Zhenguo evaluated the structural parameters and the damping parameters at different network levels [20]. However, when Rayleigh damping is employed, the physical meaning of the modes is in question. To increase the accuracy of the lightweight structure tests, the stiffness and the damping matrices are updated using an experimental setup [21]. The damping identification in Ref [22] can provide a non-proportional structural damping matrix, which can be used directly in calculations.

In this study, the computational model is updated using an analytical model, instead of an experimental one. Non-proportional structural damping and non-proportional viscous damping matrices are obtained using the response method. First, a computational and an analytical model of a 600-mm length Euler-Bernoulli aluminum beam with a rectangular cross-section of $19 \mathrm{~mm} \times 3 \mathrm{~mm}$ is constructed. The boundary conditions are fixed-free, i.e., a cantilever beam. The material properties are as follows: Young's modulus, $E=71 \mathrm{GPa}$ and density, $\rho=2,770 \mathrm{~kg} / \mathrm{m}^{3}$. Computational and analytical models are considered as continuous and discrete parameter systems. The percentage error between the computational and the analytical model is computed. In finite element modeling, as the number of elements increase, the convergence rate increases. Computational models are formed using Ansys ${ }^{\mathrm{TM}}$ where, a 1-D beam element is chosen to obtain a finite element model of the structure. Continuous and discrete parameter analytical models are constructed using Matlab ${ }^{\mathrm{TM}}$. In the first step, the eigenfrequencies and the eigenvectors of the beams are calculated by considering mass and stiffness matrices only. Computational and analytical results of the continuous and the discrete parameter models are compared for the transverse vibration case. In the second step, non-proportional structural damping and nonproportional viscous damping matrices are introduced to the analytical models. Frequency response functions of the analytical models are obtained through a state-space representation. The damping matrices are identified using the frequency response functions. The damping matrices that are used in the analytical model, and the damping matrices identified using frequency response functions are compared.

\section{Continuous Beam Model}

The continuous beam model is analyzed computationally and analytically. Transverse vibration results of the two analyses are compared. In both models, the first six eigenfrequencies are considered, and a cantilever Euler-Bernoulli aluminum beam is used. The dimensions of the beam are as follows: $\mathrm{L} \times \mathrm{W} \times \mathrm{H}=$ $600 \times 19 \times 3$ in millimeters.

\subsection{Computational Continuous Beam Model}

Ansys Workbench is employed for the computational modeling of the continuous model. A 1-D beam element is used for the modeling procedure. In the first step, an undamped model is constructed. The beam is modeled in different element numbers to observe the convergence in the results. Convergence in the results is tabulated in Table 1 , where the number of elements is increased systematically. 


\subsection{Analytical Continuous Beam Model}

The analytical solution of the continuous beam model

$$
\frac{d^{2}}{d x^{2}}\left\{\operatorname{EI}(x) \frac{d^{2} Y(x)}{x^{2}}\right\}=\omega^{2} m(x) Y(x)
$$
is obtained using Matlab 2019b. The equation of motion for a continuous Euler-Bernoulli beam is given as follows:

Table 1 The comparison of first six eigenfrequencies of computational continuous model and analytical continuous model

\begin{tabular}{|c|c|c|c|c|c|c|c|c|c|}
\hline & \multirow{2}{*}{$\begin{array}{c}\text { Analytical } \\
\text { continuous mode } \\
\text { frequencies (Hz) }\end{array}$} & \multicolumn{9}{|c|}{ Computational continuous mode frequencies (Hz) } \\
\cline { 3 - 13 }$n$ & 6 Elements & Error (\%) & 8 Elements & Error (\%) & 10 Elements & Error (\%) & 100 Elements & Error (\%) \\
\hline 1 & 6,815 & 6,815 & 0,0 & 6,815 & 0,0 & 6,815 & 0,0 & 6,815 & 0,0 \\
\hline 2 & 42,711 & 42,738 & 0,06 & 42,715 & 0,01 & 42,709 & 0,005 & 42,705 & 0,01 \\
\hline 3 & 119,592 & 120,24 & 0,54 & 119,77 & 0,15 & 119,64 & 0,04 & 119,55 & 0,04 \\
\hline 4 & 234,353 & 239,13 & 2,04 & 235,82 & 0,63 & 234,89 & 0,23 & 234,21 & 0,06 \\
\hline 5 & 387,402 & 408,46 & 5,44 & 394,04 & 1,71 & 389,99 & 0,67 & 387,02 & 0,1 \\
\hline 6 & 578,712 & 655,05 & 13,20 & 600,48 & 3,76 & 587,47 & 1,51 & 577,88 & 0,14 \\
\hline
\end{tabular}

In Eq. (1), $E$ is the modulus of elasticity, $I$ is the moment of inertia of the beam's cross section, $Y(x)$ is transverse displacement, $\omega$ is natural frequency, $m$ is mass per unit length, and $x$ is the distance from the fixed point of the beam (See A.1 for a closed form solution).

Computationally and analytically calculated eigenfrequencies are tabulated in Table 1, and the percentage error is calculated over the analytical results. The third mode shape of the continuous six dof beam model is shown in Figure 1.

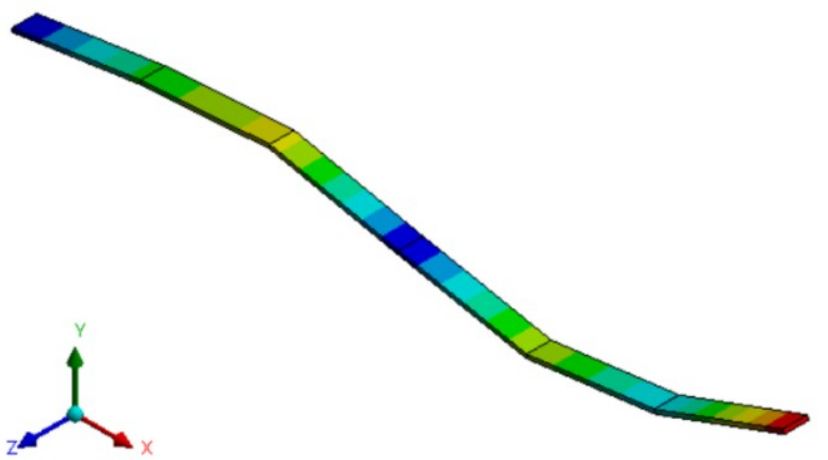

Figure 1. Third mode shape of the computational continuous model

\section{Discrete Beam Model}

For discrete parameter modeling, the identical beam is analyzed computationally and analytically. Computational discrete beam model analysis results are obtained using Ansys APDL Mechanical, and Matlab is employed to construct the analytical discrete parameter model. Eigenfrequencies of the 6-element beam are calculated. In the first step, the results of an undamped model are compared. Then, the frequency response functions are generated by assigning non-proportional damping values to the analytical discrete parameter model. The damping matrices are identified through the generated frequency response functions. (See Section 5)

\subsection{Computational Discrete Beam Model}

A 6-element beam model is constructed in Ansys APDL Mechanical, and the eigenfrequencies are computed. The computed eigenfrequencies are tabulated in Table 2. First mode shape of the computational discrete parameter model is shown in Figure 2.

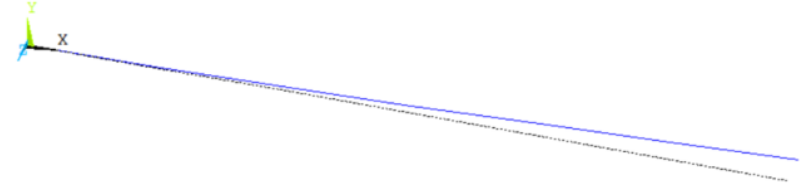

Figure 2. First mode shape of the computational discrete parameter model

\subsection{Analytical Discrete Beam Model}

The 6-dof discrete parameter model is built in Matlab using a state-space representation (See A.2). The 6-dof analytical discrete parameter model is shown in Figure 3, where $m, k, c$ and $d$ stand for mass, stiffness, viscous damping, and structural damping, respectively. Eigenfrequencies and frequency response functions can be calculated by introducing mass, stiffness, and damping matrices to the system. The mass and stiffness matrices are 
derived from Ansys APDL Mechanical using the "DMAT" command. The mass matrix and the stiffness matrix are given in Table 3 and Table 4, respectively. The undamped eigenfrequencies and the percentage error values are tabulated in Table 2. Then, viscous, and structural damping matrices, where introduced in Section 5 are assigned.

Table 2. Comparison of eigenfrequencies of computational discrete beam model and analytical discrete beam model.

\begin{tabular}{llll}
\hline Mode & $\begin{array}{l}\text { Computational- } \\
\text { discrete }(\mathrm{rad} / \mathrm{s})\end{array}$ & $\begin{array}{l}\text { Analytical- } \\
\text { discrete }(\mathrm{rad} / \mathrm{s})\end{array}$ & Error (\%) \\
\hline 1 & 891,08 & 891,23 & 0,02 \\
\hline 2 & 2734,57 & 2735 & 0,02 \\
\hline 3 & 4762,15 & 4762,96 & 0,02 \\
\hline 4 & 7068,58 & 7069,94 & 0,02 \\
\hline 5 & 9552,95 & 9554,43 & 0,02 \\
\hline 6 & 11463,04 & 11464,73 & 0,02 \\
\hline
\end{tabular}

Table 3. The mass matrix of analytical discrete beam model

\begin{tabular}{cccccc}
\hline $1.053 \mathrm{e}-2$ & $2.631 \mathrm{e}-3$ & 0 & 0 & 0 & 0 \\
$2.631 \mathrm{e}-3$ & $1.053 \mathrm{e}-2$ & $2.631 \mathrm{e}-3$ & 0 & 0 & 0 \\
0 & $2.631 \mathrm{e}-3$ & $1.053 \mathrm{e}-2$ & $2.631 \mathrm{e}-3$ & 0 & 0 \\
0 & 0 & $2.631 \mathrm{e}-3$ & $1.053 \mathrm{e}-2$ & $2.631 \mathrm{e}-3$ & 0 \\
0 & 0 & 0 & $2.631 \mathrm{e}-3$ & $1.053 \mathrm{e}-2$ & $2.631 \mathrm{e}-3$ \\
0 & 0 & 0 & 0 & $2.631 \mathrm{e}-3$ & $5.263 \mathrm{e}-3$ \\
\hline
\end{tabular}

Table 4. The stiffness matrix of analytical discrete beam model

\begin{tabular}{rrrrrr}
\hline $3.637 \mathrm{e} 5$ & $-1.819 \mathrm{e} 5$ & 0 & 0 & 0 & 0 \\
$-1.819 \mathrm{e} 5$ & $3.637 \mathrm{e} 5$ & $-1.819 \mathrm{e} 5$ & 0 & 0 & 0 \\
0 & $-1.819 \mathrm{e} 5$ & $3.637 \mathrm{e} 5$ & $-1.819 \mathrm{e} 5$ & 0 & 0 \\
0 & 0 & $-1.819 \mathrm{e} 5$ & $3.637 \mathrm{e} 5$ & $-1.819 \mathrm{e} 5$ & 0 \\
0 & 0 & 0 & $-1.819 \mathrm{e} 5$ & $3.637 \mathrm{e} 5$ & $-1.819 \mathrm{e} 5$ \\
0 & 0 & 0 & 0 & $-1.819 \mathrm{e} 5$ & $1.819 \mathrm{e} 5$ \\
\hline
\end{tabular}

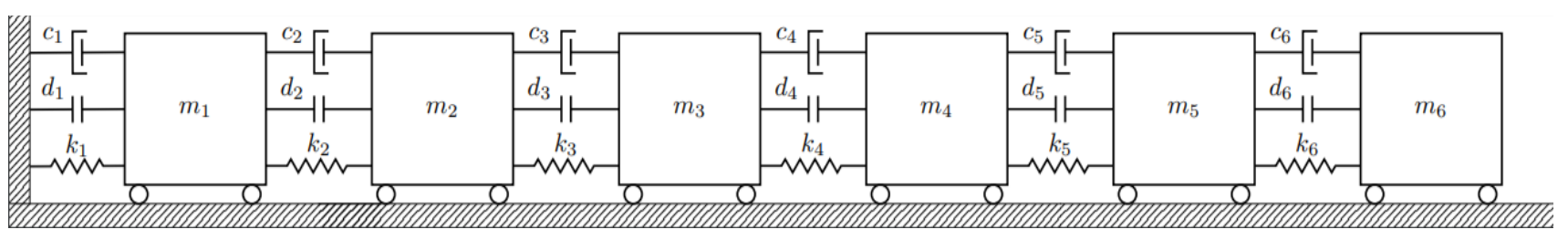

Figure 3 Discrete parameter system of six degrees of freedom analytical model (m: mass; k: stiffness, c: viscous damping; d: structural damping)

\section{Damping Identification}

Equation of motion of a damped system in time domain is given as follows:

$\mathbf{M} \ddot{\boldsymbol{x}}(t)+\mathbf{C} \dot{\boldsymbol{x}}(t)+(i \mathbf{D}+\mathbf{K}) \boldsymbol{x}(t)=\boldsymbol{f}(t)$

Here $\boldsymbol{M}, \boldsymbol{K}, \boldsymbol{C}$ and $\boldsymbol{D}$ represent $\mathrm{n} \times \mathrm{n}$ mass, stiffness, viscous damping, and structural damping matrices, respectively. $\boldsymbol{f}$ and $\boldsymbol{x}$ are $n \times 1$ force and displacement vectors. If the excitation is harmonic, $f(t)=\mathrm{F}(\omega) e^{i \omega t}$ and $x(t)=\mathrm{X}(\omega) e^{i \omega t}$. Hence, Eq. (2) can be written in frequency domain as follows:

$\left[-\omega^{2} \mathbf{M}+i \mathbf{C} \omega+i \mathbf{D}+\mathbf{K}\right] \mathbf{X}(\omega)=\mathbf{F}(\omega)$

The term in square brackets is the dynamic stiffness matrix, and the inverse of this matrix is the complex receptance matrix $\left(\mathrm{R}^{c}(\omega)\right)$ :

$\left[\left(\mathbf{K}-\omega^{2} \mathbf{M}\right)+i(\mathbf{C} \omega+\mathbf{D})\right]=\left[\mathbf{R}^{c}(\omega)\right]^{-1}$

The receptance matrix can be populated using the receptance functions estimated through experimentation, where the displacement vector $\boldsymbol{X}(\omega)$ is measured. The relation is given by:

$\mathbf{X}(\omega)=\mathbf{R}^{c}(\omega) \mathbf{F}(\omega)$

The complex receptance matrix consists of real and imaginary parts, which can be expressed:

$\mathbf{R}^{c}(\omega)=\boldsymbol{R}_{\boldsymbol{R}}^{c}(\omega)+i \boldsymbol{R}_{I}^{c}(\omega)$

where the subscripts $R$ and $I$ stand for the real and imaginary values of the matrix, respectively. The undamped or normal receptance matrix can be expressed as follows:

$\mathbf{R}^{N}(\omega)=\left[\mathbf{K}-\omega^{2} \mathbf{M}\right]^{-1}$

which is the receptance type frequency response function matrix generated from the normal modes. Pre-multiplying Eq. (2) by the normal receptance matrix yield

$\mathbf{X}(\omega)+i \mathbf{R}^{N}(\omega)(\omega \mathbf{C}+\mathbf{D}) \mathbf{X}(\omega)=\mathbf{R}^{N}(\omega) \mathbf{F}(\omega)$ 
Transformation matrix can be expressed by

$\mathbf{T}(\omega)=\mathbf{R}^{N}(\omega)(\omega \mathbf{C}+\mathbf{D})$

Substituting the transformation matrix into Eq. (8), yields

$[\mathbf{I}+i \mathbf{T}(\omega)] \mathbf{X}(\omega)=\mathbf{R}^{N}(\omega) \mathbf{F}(\omega)$

where I denote identity matrix. Using the relation given by Eq. (6), Eq. (10) can be rewritten as

$[\mathbf{I}+i \mathbf{T}(\omega)] \mathbf{R}^{c}(\omega)=\mathbf{R}^{N}(\omega)$

so as to eliminate the displacement vector $\mathbf{X}(\omega)$. Substituting Eq. (6) into Eq. (11) yields

$[\mathbf{I}+i \mathbf{T}(\omega)]\left[\mathbf{R}_{R}^{c}(\omega)+i \mathbf{R}_{I}^{c}(\omega)\right]=\mathbf{R}^{N}(\omega)$

which can be rearranged as

$$
\begin{aligned}
{\left[\mathbf{R}_{R}^{c}(\omega)-\mathbf{T}(\omega) \mathbf{R}_{I}^{c}(\omega)\right] } & \\
& +i\left[\mathbf{R}_{I}^{c}(\omega)\right. \\
& \left.+\mathbf{T}(\omega) \mathbf{R}_{I}^{c}(\omega)\right]=\mathbf{R}^{N}(\omega)
\end{aligned}
$$

where the left-hand side has real and imaginary components. Since the right-hand side of Eq. (13) has only real components, the following statements are true for all frequencies:

$$
\begin{aligned}
& {\left[\mathbf{R}_{I}^{c}(\omega)+\mathbf{T}(\omega) \mathbf{R}_{R}^{c}(\omega)\right]=0 \rightarrow} \\
& \mathbf{T}(\omega)=-\mathbf{R}_{I}^{c}(\omega)\left[\mathbf{R}_{R}^{c}(\omega)\right]^{-1} \\
& \mathbf{R}_{R}^{c}(\omega)-\mathbf{T}(\omega) \mathbf{R}_{I}^{c}(\omega)=\mathbf{R}^{N}(\omega)
\end{aligned}
$$

Viscous and structural damping can be identified by substituting Eq. (9) into Eq. (14), which yields:

$(\omega \mathbf{C}+\mathbf{D})=-\left[\mathbf{R}^{N}(\omega)\right]^{-1} \mathbf{R}_{I}^{c}(\omega)\left[\mathbf{R}_{R}^{c}(\omega)\right]^{-1}$

If the complex receptance matrix has been measured experimentally, the normal receptance matrix can be calculated, and if the normal receptance matrices are known, damping matrix can be calculated. Note that, Eq. (16) can be solved in a least-squares approach. It is recommended to use the pseudo inverse operator $([\cdot]+)$ for the matrix inversion, which returns a least-squares solution. Hence,

$$
\left[\begin{array}{l}
\mathbf{D} \\
\mathbf{C}
\end{array}\right]=-\left[\begin{array}{cc}
\mathbf{R}^{N} & \omega_{1} \mathbf{R}^{N} \\
\vdots & \vdots \\
\mathbf{R}^{N} & \omega_{n} \mathbf{R}^{N}
\end{array}\right]^{+}\left[\begin{array}{c}
\mathbf{R}_{I}^{c}\left(\omega_{1}\right) \\
\vdots \\
\mathbf{R}_{I}^{c}\left(\omega_{n}\right)
\end{array}\right]\left[\begin{array}{c}
\left(\mathbf{R}_{R}^{c}\left(\omega_{1}\right)\right)^{+} \\
\vdots \\
\left(\mathbf{R}_{R}^{c}\left(\omega_{n}\right)\right)^{+}
\end{array}\right]
$$

\section{Frequency Response Functions}

In this section, non-proportional damping matrices are assigned to the analytical discrete parameter model, and the complex frequency response functions are generated. The assigned viscous and structural damping matrices are identified through the complex frequency response functions.

One of the frequency response functions of the nonproportional structural damped six degrees of freedom discrete parameter model, namely FRF11 is shown in Figure 4. In Figure 5, all the diagonal terms of the frequency response function matrix of the discrete parameter model are given. To yield the effect of damping on the model, the undamped aka normal frequency response functions (NFRFs) of the model are compared with the damped frequency response function. The comparisons are made for viscously damped and structurally damped models in Figures 6 and 7, respectively.

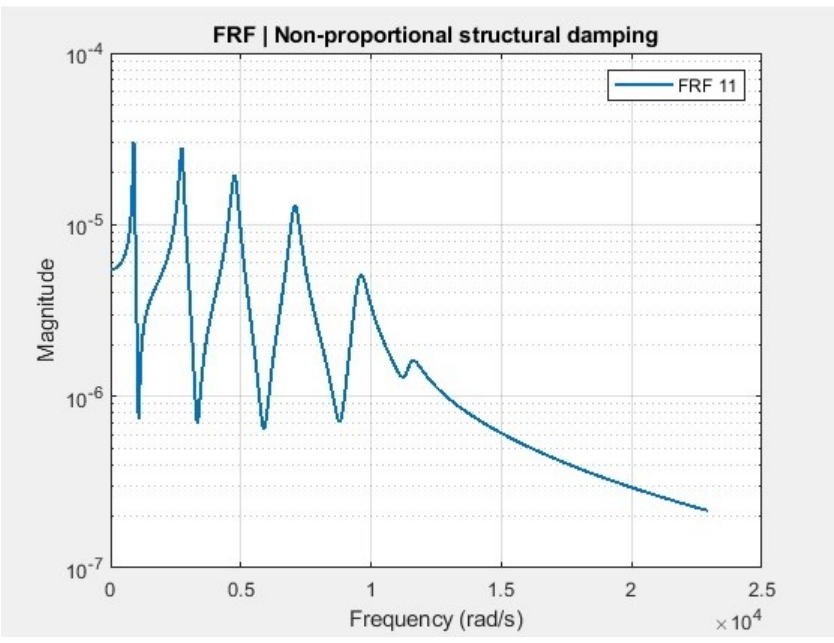

Figure 4. Frequency response function of the nonproportional structural damped six degrees of freedom discrete parameter model (FRF 11)

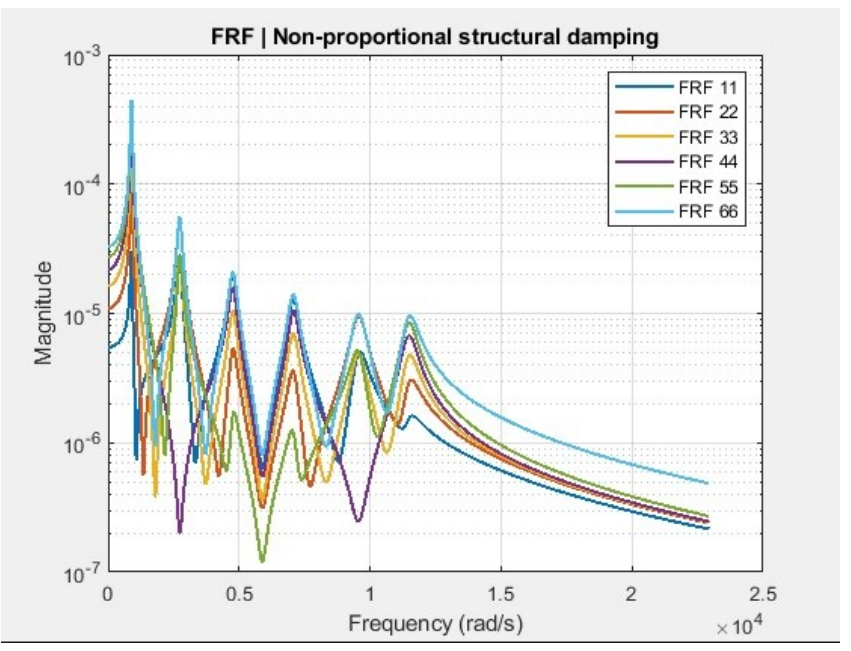

Figure 5. Frequency response functions of nonproportional structural damped six degrees of freedom discrete parameter model (FRFs) 


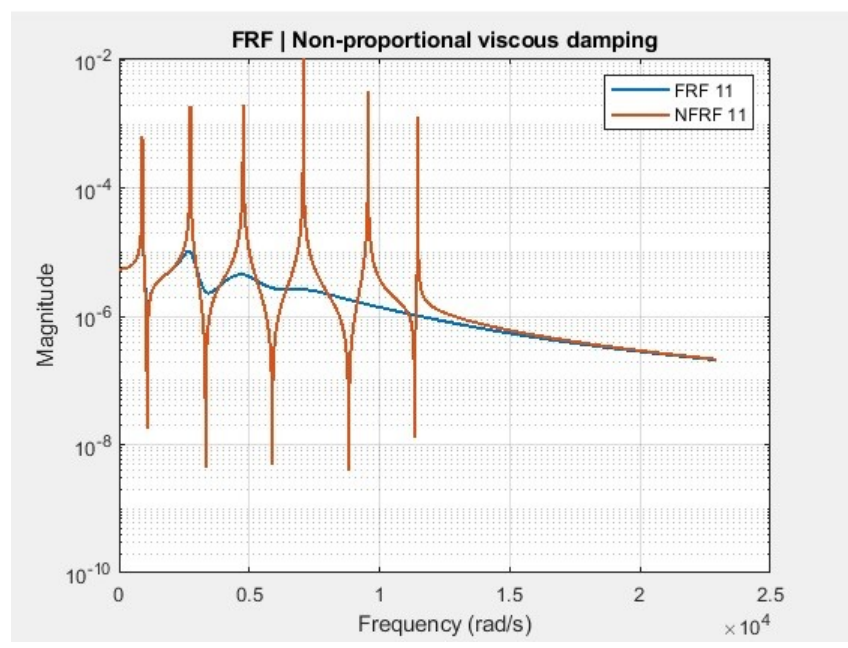

Figure 6. Frequency response function and normal frequency response function of the non-proportional viscous damped six degrees of freedom discrete parameter model (FRF 11 and NFRF 11)

The non-proportional viscous damping matrix assigned to the analytical discrete parameter model is as follows:

$$
\mathbf{C}=\left[\begin{array}{cccccc}
18 & -10 & 0 & 0 & 0 & 0 \\
-10 & 25 & -15 & 0 & 0 & 0 \\
0 & -15 & 27 & -12 & 0 & 0 \\
0 & 0 & -12 & 26 & -14 & 0 \\
0 & 0 & 0 & -14 & 27 & -13 \\
0 & 0 & 0 & 0 & -13 & 13
\end{array}\right]
$$

The identified viscous damping matrix through the complex frequency response functions is as follows:

$\mathbf{C}_{\text {identified }}=\left[\begin{array}{cccccc}18 & -10 & 0 & 0 & 0 & 0 \\ -10 & 25 & -15 & 0 & 0 & 0 \\ 0 & -15 & 27 & -12 & 0 & 0 \\ 0 & 0 & -12 & 26 & -14 & 0 \\ 0 & 0 & 0 & -14 & 27 & -13 \\ 0 & 0 & 0 & 0 & -13 & 13\end{array}\right]$

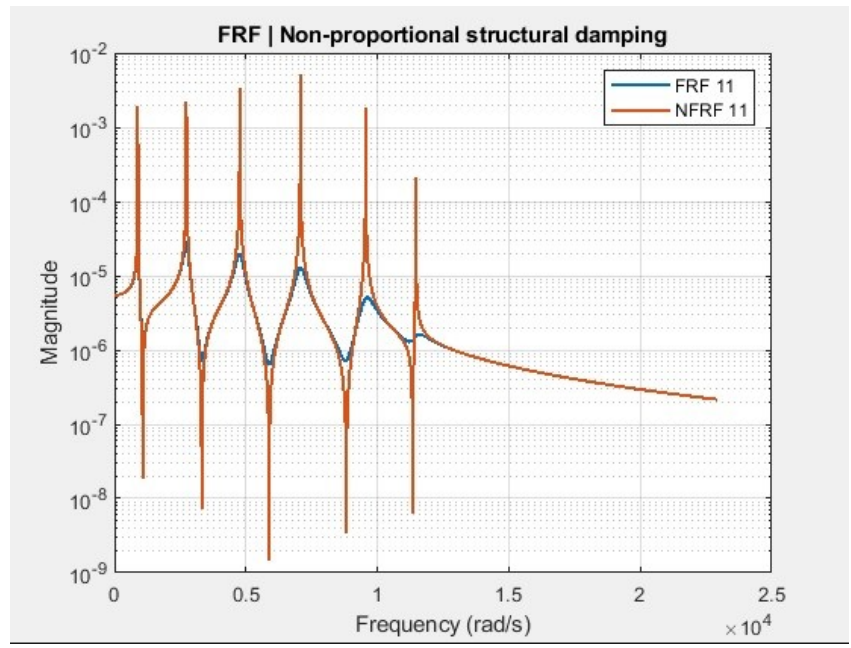

Figure 7. Frequency response function and normal frequency response function of the non-proportional structural damped six degrees of freedom discrete parameter model (FRF 11 and NFRF 11)
The non-proportional structural damping matrix assigned to the analytical discrete parameter model is as follows:

$$
\begin{gathered}
\mathbf{D}= \\
{\left[\begin{array}{cccccc}
2382,9 & -11095,9 & 0 & 0 & 0 & 0 \\
-11095,9 & 21646,1 & -10550,2 & 0 & 0 & 0 \\
0 & -10550,2 & 17989,91 & -7439,71 & 0 & 0 \\
0 & 0 & -7439,71 & 18353,71 & -10914 & 0 \\
0 & 0 & 0 & -10914 & 20009 & -9095 \\
0 & 0 & 0 & 0 & -9095 & 9095
\end{array}\right]}
\end{gathered}
$$

The identified structural damping matrix through the complex frequency response functions is as follows:

$$
\mathbf{D}_{\text {identified }}=
$$

$\left[\begin{array}{cccccc}2382,9 & -11095,9 & 0 & 0 & 0 & 0 \\ -11095,9 & 21646,1 & -10550,2 & 0 & 0 & 0 \\ 0 & -10550,2 & 17989,91 & -7439,71 & 0 & 0 \\ 0 & 0 & -7439,71 & 18353,71 & -10914 & 0 \\ 0 & 0 & 0 & -10914 & 20009 & -9095 \\ 0 & 0 & 0 & 0 & -9095 & 9095\end{array}\right]$

It is shown that the assigned and the identified damping matrices are identical.

\section{Conclusions}

- It is shown that the damping matrix values assigned to the model can be identified identically through the complex frequency response functions of the model.

- Once the frequency response functions of a structure are known by measurement, the structural and viscous damping matrices can be identified.

- If the computational model is updated using the identified damping matrices, the results will be more accurate for engineering structures.

\section{Conflict of Interests}

No conflict of interest was stated by the authors.

\section{Declaration of Ethical Standards}

The authors of this article declares that the materials and methods used in this study do not require ethical committee permission and legal-special permission.

\section{References}

[1] Mottershead J.E., Friswell M.I., 1993. Model updating in structural dynamics: a survey. Journal of Sound and Vibration, 167, 347-375. 
[2] Baruch M., 1978. Optimization procedure to correct stiffness and flexibility matrices using vibration tests. AIAA Journal, 16, 1208-1210.

[3] Berman A., Nagy E.J., 1983. Improvement of a large analytical model using test data. AIAA Journal, 21, 1168-1173.

[4] Wei F-S., 1990. Analytical dynamic model improvement using vibration test data. AIAA Journal, 28, $175-177$.

[5] Friswell M.I., Inman D., Pilkey D., 1998. Direct updating of damping and stiffness matrices. AIAA Journal, 36, 491-493.

[6] Imregun M., Visser W.J., Ewins D.J., 1995. Finite element model updating using frequency response function data -1 : Theory and initial investigation. Journal of Mechanical Science and Technology, 9, 187-202.

[7] Lin R-M., Ewins D., 1990. Model updating using FR data. Paper presented at the $15^{\text {th }}$ International Seminar on Modal Analysis, pp.141-162.

[8] Kwon K.S., Lin R.M., 2005. Robust finite element model updating Taguchi method. Journal of Sound and Vibration, 280, 77-99.

[9] Jacquelin E., Adhikari S., Fiswell M.I., 2012. A second-moment approach for direct probabilistic model updating in structural dynamics. Mechanical Systems and Signal Processing, 29, 262-283.

[10] Sipple J.D., Sanayei M., 2014. Finite element model updating using frequency response functions and numerical sensitivities. Structural Control and Health Monitoring, 21, 784-802.

[11] Pradhan S., Modak S., 2012. Normal response function method for mass and stiffness matrix updating using complex FRFs. Mechanical Systems and Signal Processing, 32, 232-250.

[12] Arora V., 2014. FE model updating method incorporating damping matrices for structural dynamic modifications. Structural Engineering and Mechanics, 52, 261-274.

[13] Matta E., Stefano A., 2012. Robust finite element model updating of a large-scale benchmark building structure. Structural Engineering and Mechanics, 43, 371-394.

[14] Adhikari S., 2001. Damping models for structural vibration. Doctoral dissertation, University of Cambridge, Cambridge, England.
[15] Salamon R., Kamiński H., Fritzkowski P., 2020. Estimation of parameters of various damping models in planar motion of a pendulum. Meccanica, 1-23.

[16] Shadan F., Khoshnoudian F., Inman D.J., Esfandiari A., 2018. Experimental validation of a FRF-based model updating method. Journal of Vibration and Control, 24, 1570-1583.

[17] Shadan F., Khoshnoudian F., Esfandiari A., 2016. A frequency response- based structural damage identification using model updating method. Structural Control and Health Monitoring, 23, 286302.

[18] Zin M.M., Rani M.A., Yunus M.A., Sani M.S.M., Wan Iskandar Mirza W.I.I., Mat Isa A.A. 2018. Frequency response function (FRF) based updating of a laser spot welded structure. In AIP Conference Proceedings (Vol. 1952, No. 1, p. 020055). AIP Publishing LLC.

[19] Natke H.G., 1988. Updating computational models in the frequency domain based on measured data: a survey. Probabilistic Engineering Mechanics, 3, 2835 .

[20] Lu Y., Zhenguo T., 2004. A two-level neural network approach for dynamic FE model updating including damping. Journal of Sound and Vibration, 275, 931952.

[21] Lepoittevin G., Gerald K., 2011. Finite element model updating of vibrating structures under freefree boundary conditions for modal damping prediction., Mechanical Systems and Signal Processing, 25, 2203-2218.

[22] Oktav A., 2020. Identification of non-proportional structural damping using experimental modal analysis data. Journal of Measurements in Engineering, 8, 3445 .

\section{Appendices}

Using (Eq.1) and the boundary conditions for a cantilever beam, the closed form natural frequency can be written as follows:

$$
\begin{aligned}
\omega_{i} & =\beta_{i}^{2} \sqrt{\frac{E I}{m L^{4}}} \\
\beta_{i} & =1.875,4.694,7.885, . .
\end{aligned}
$$


The state-space representation used for the system is as follows:

$$
\begin{aligned}
& \boldsymbol{A}=\left[\begin{array}{cc}
\mathbf{0} & \boldsymbol{I} \\
-\boldsymbol{M}^{-1}(i \boldsymbol{D}+\boldsymbol{K}) & -\boldsymbol{M}^{-1} \boldsymbol{C}
\end{array}\right] \\
& \boldsymbol{B}=\left[\begin{array}{c}
\mathbf{0} \\
\boldsymbol{M}^{-1}
\end{array}\right] ; \quad \boldsymbol{C}=\left[\begin{array}{ll}
\boldsymbol{I} & \mathbf{0}
\end{array}\right] ; \quad \boldsymbol{D}=\mathbf{0}
\end{aligned}
$$

